\title{
All-solid-state Reference Electrodes for analytical applications
}

\author{
Francesca Criscuolo ${ }^{*, \S}$, Marco Galfione ${ }^{\S}$, Sandro Carrara, Giovanni De Micheli \\ ${ }^{a}$ Swiss Federal Institute of Technology (EPFL), Lausanne, Switzerland \\ $\S$ These authors contributed equally to this work. \\ *Email: francesca.criscuolo@epfl.ch
}

\begin{abstract}
The increasing development of electrochemical wearable and Lab-On-a-Chip (LOC) sensors have created the urgent need of efficient and stable miniaturized Reference Electrodes (REs). Ag/AgCl REs are the simplest, most common and environmental friendly REs. Conventional liquid-filled REs are not suitable for the portable applications because of the difficult trapping of the solution in miniaturized electrodes and of the high sensitivity to temperature and pressure variations. In recent years several attempts to build all-solid-state REs have been proposed in literature. However, many challenges still need to be overcome before reaching the commercialization stage, including the continuous leaching of the loaded ions and the influence of the sample solution on the interfacial potential. In this paper, we investigate and compare different routes to fabricate solidcontacts REs. The produced REs are used for the calibration of nanostructured potentiometric sensors. The different stability at various chlorides concentrations and $\mathrm{pH}$ is also investigated. Finally, their voltammetric response is compared to a Double Junction (DJ) RE.
\end{abstract}

Index Terms-Reference Electrode; Electrochemistry; Potentiometry; Cyclic Voltammetry.

\section{INTRODUCTION}

The past few years has seen a growing interest towards portable and especially wearable electrochemical systems. In particular, all-solid-state potentiometric ion-sensors have been extensively used for environmental monitoring and for measuring the concentration of electrolytes in different body fluids for personalized healthcare. However, the realization of adequate and stable Reference Electrodes (REs) still represents one of the most challenging issues that prevents their commercialization. Although various strategies have been proposed in literature, this problem is still far from a final solution [1], [2].

The $\mathrm{Ag} / \mathrm{AgCl}$ double junction $\mathrm{RE}$ is the most commonly used type of RE thanks to its excellent stability, simplicity and non-toxicity. [1] Due to its popularity, it is the most targeted $\mathrm{RE}$ for miniaturized systems. In particular, the realization of all-solid-state $\mathrm{Ag} / \mathrm{AgCl} \mathrm{REs}$ represents one of the most attractive strategies since the elimination of the liquid junction eliminates problems of evaporation or sensitivity to temperature and pressure variations [3], [4] . A solid-state exchange membrane doped with $\mathrm{KCl}$ (or $\mathrm{NaCl} / \mathrm{AgCl}$ ) is used to replace the liquid junction of conventional REs. Different strategies have been proposed (Fig. 1), which include the impregnation of a polymer with ionic liquids [5], [6], solid doping of agar [7], [8] or of a polymeric membrane [9], [10].
1) SOLID KCI DOPING

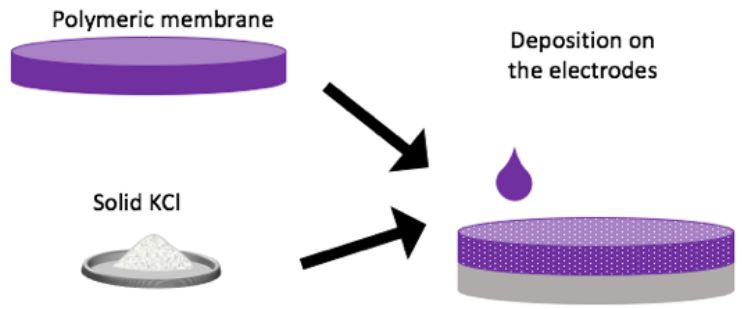

2) IMPREGNATION OF A POLYMER

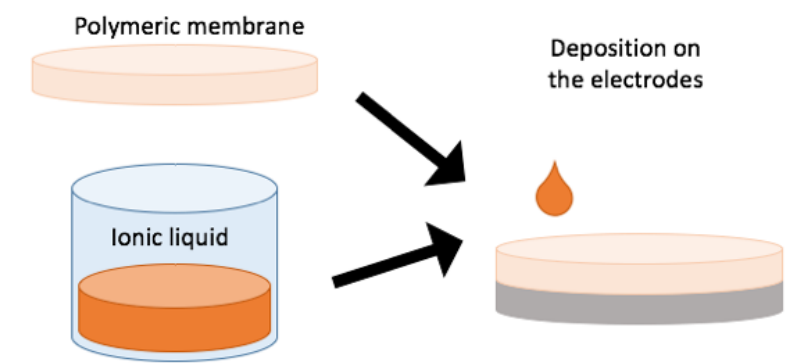

3) AGAR DOPING
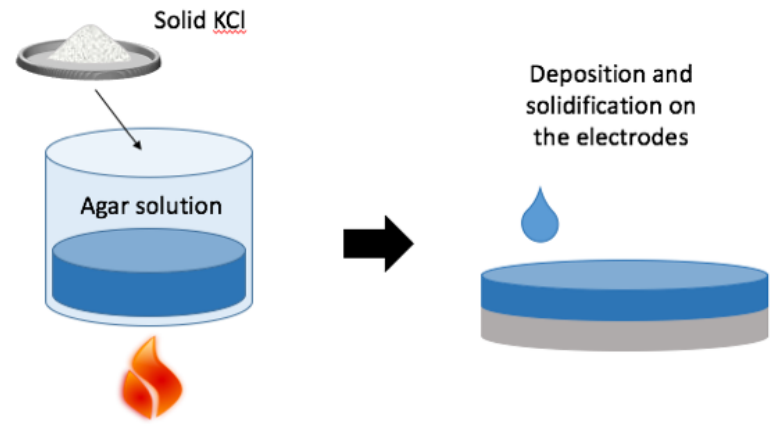

Fig. 1. Different strategies for the fabrication of reference membranes.

In this paper, we produce different REs membranes to characterize and compare their electrochemical behaviour in different conditions: Agar, Polyvinyl Butyral (PVB), Polyvinyl Chloride (PVC), Ionic-Liquid(IL)-doped PVC. The membranes are then used to calibrate lithium Ion-Selective Elec- 
TABLE I

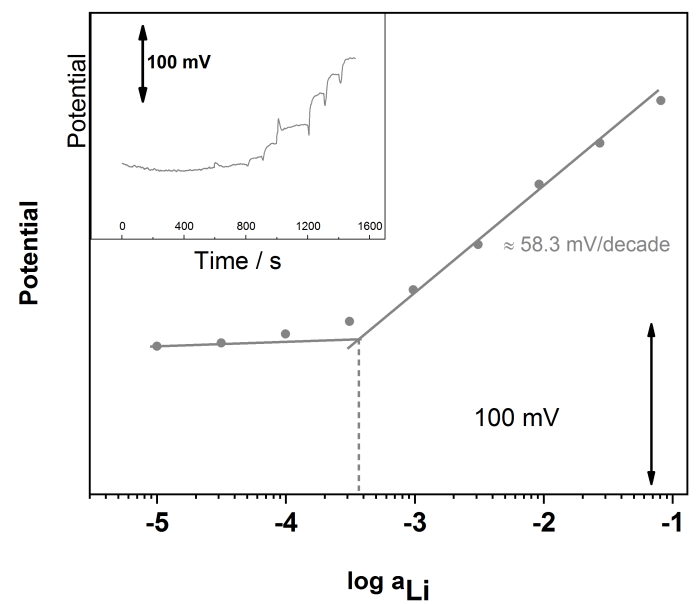

Fig. 2. Example of a calibration of a $\mathrm{Li}^{+}$ISE with a all-solid-state $\mathrm{RE}$ based on a IL-PVC membrane. The corresponding calibration time trace is given in the small inset.

trodes (ISEs). The influence of chloride concentration and of $\mathrm{pH}$ on their stability is investigated to help defining the best membrane for different applications.

\section{EXPERIMENTAL METHODS}

\section{A. Materials}

All chemicals were obtained from Sigma Aldrich. Silver, platinum and gold screen-printed electrodes with a diameter of $4 \mathrm{~mm}$ were obtained from DropSens (Spain).

\section{B. Electrode fabrication}

The chemical chlorination was performed in a $50 \mathrm{mM} \mathrm{FeCl}_{3}$ solution for 3 minutes.

The agar membrane was produced with $0.8 \mathrm{~g}$ of agar in 50 $\mathrm{ml}$ of $1 \mathrm{M} \mathrm{KCl}$. After reaching the boiling temperature, the solution was cooled to $65 \mathrm{C}$ and then spread over the electrode. In all other cases 10 ul of membrane solution was drop-cast on the electrodes. The membrane cocktail compositions are the folowing: $79.1 \mathrm{mg}$ of $\mathrm{PVB}$ and $50 \mathrm{mg}$ of $\mathrm{NaCl}$ in ethanol for PVB; $0.4 \mathrm{~g}$ of $\mathrm{PVC}$ in $\mathrm{NaCl}$ saturated Tetrahydrofuran (THF) for PVC; $0.1 \%$ of IL, $33 \%$ of PVC powder, 66 $\%$ of Bis(2-ethylhexyl) sebacate in $0.5 \mathrm{~mL}$ of THF for the PVC membrane doped with the ionic liquid. 5 replicas were produced per sample type.

$\mathrm{Li}^{+}$ISEs with nanostructured solid-contacts were fabricated as described elsewhere [11]. The calibrations were performed by subsequent $\mathrm{LiCl}$ additions.

\section{Morphological and electrochemical characterization}

A two-electrodes setup was used in all potentiometric measurements using a Double Junction (DJ) $\mathrm{Ag} / \mathrm{AgCl} \mathrm{RE}$ from Metrohm filled with $3 \mathrm{M} \mathrm{KCl}$ and lithium acetate as internal and external solutions, respectively. Cyclic Voltammetry (CV) was performed in a three-electrodes setup. SEM images were obtained with a Merlin microscope from Zeiss in SE mode using an In Lens detector.
SENSORS PARAMETERS OF A Li ${ }^{+}$ISE OBTAINED WITH DIFFERENT RES.

\begin{tabular}{|c|c|c|}
\hline & $\begin{array}{c}\text { sensitivity } \\
{[\mathrm{mV} / \text { decade }]}\end{array}$ & $\begin{array}{c}\text { LOD } \\
(\mathrm{M})\end{array}$ \\
\hline No membrane & $\mathbf{1 0 8 . 8} \pm 31.6$ & $\mathbf{( 3 . 9 7} \pm 3.65) \times 10^{-4}$ \\
PVB & $\mathbf{6 3 . 9} \pm 13.0$ & $(\mathbf{2 . 3 0} \pm 1.57) \times 10^{-4}$ \\
Agar & $\mathbf{1 1 2 . 9} \pm 24.8$ & $(\mathbf{4 . 0 7} \pm 3.52) \times 10^{-4}$ \\
PVC & $\mathbf{5 4 . 5} \pm 9.5$ & $(\mathbf{1 . 1} \pm 0.62) \times 10^{-4}$ \\
IL-PVC & $\mathbf{5 7 . 7} \pm 0.9$ & $(\mathbf{1 . 1} \pm 0.27) \times 10^{-4}$ \\
DJ & $\mathbf{5 8 . 7} \pm 0.8$ & $(\mathbf{0 . 7 2} \pm 0.25) \times 10^{-4}$ \\
\hline
\end{tabular}
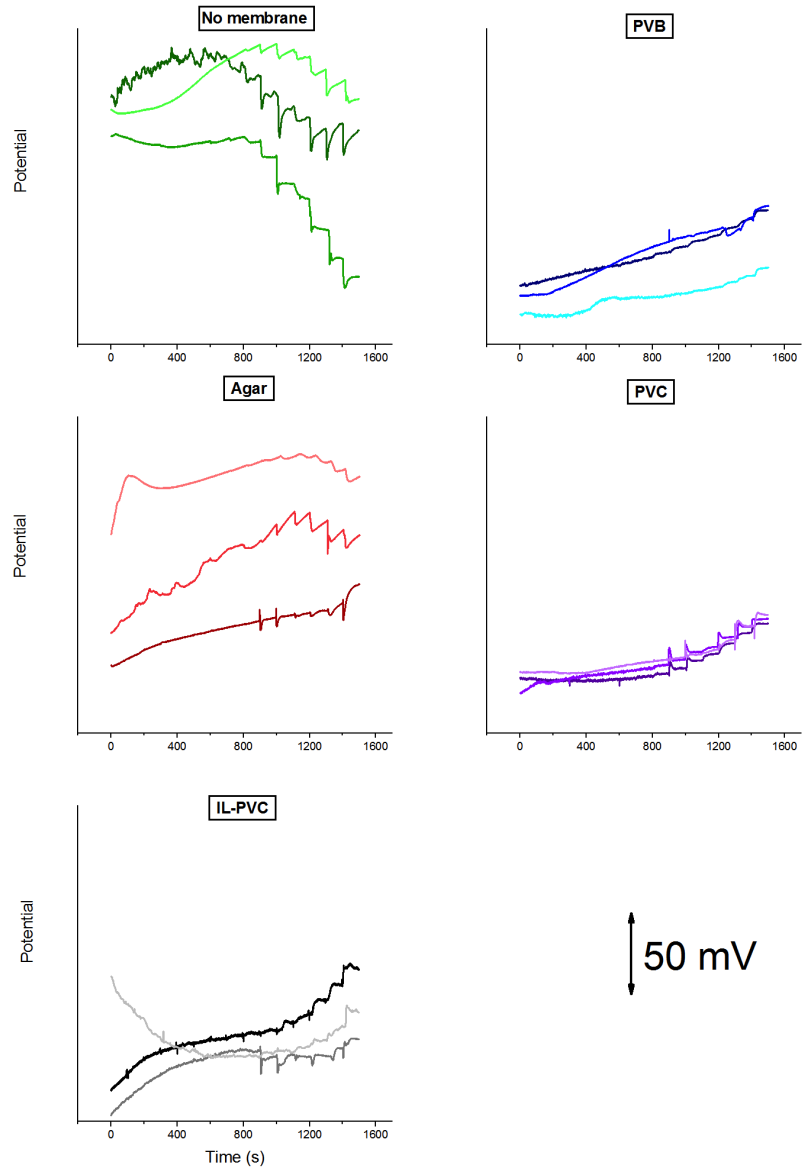

$50 \mathrm{mV}$

Fig. 3. Dependence of RE potential at increasing chloride concentrations from $10^{-9}$ up to $10^{-1} \mathrm{M}$ by subsequent chloride additions every 100 s (activity steps of a decade up to $10^{-6} \mathrm{M}$, half a decade up to $10^{-1} \mathrm{M}$ ). The responses of three different samples are reported per each RE type.

\section{EXPERIMENTAL RESULTS AND DISCUSSION}

Different ion-exchange reference membranes are proposed and compared in this work to improve the performance of simple solid-state $\mathrm{Ag} / \mathrm{AgCl}$ REs: solid-doped agar, solid-doped PVB, solid-doped PVC and IL-doped PVC.

The fabricated REs are tested for the calibration of $\mathrm{Li}^{+}$ISEs based on nanostructured solid-contacts. A typical calibration example is given in Figure 2 for a IL-PVC RE. All calibrations are performed by subsequent $\mathrm{LiCl}$ additions. All sensors pa- 

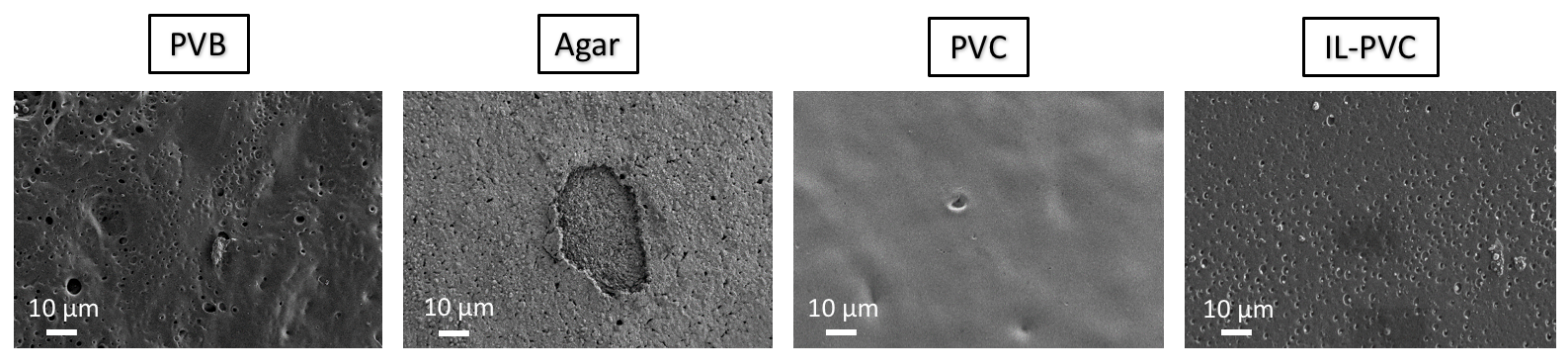

Fig. 4. SEM images of the different reference membranes.
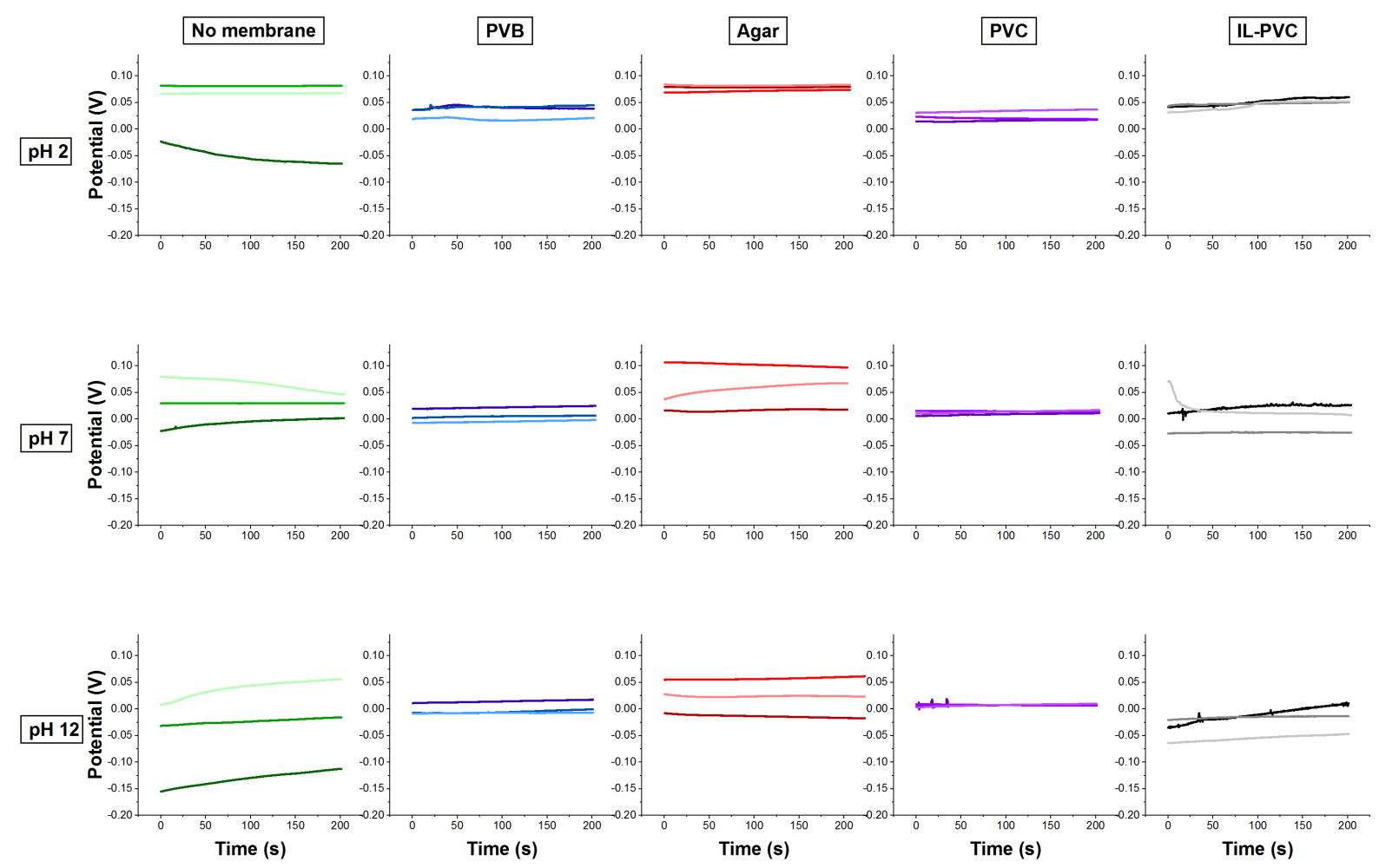

Fig. 5. Reference membranes potential at different $\mathrm{pH}$. The responses of three different samples are reported per each $\mathrm{RE}$ type.

rameters obtained using different all-solid-state REs are given in Table I in comparison with a conventional DJ $\mathrm{Ag} / \mathrm{AgCl} \mathrm{RE}$ and with a chlorinated planar $\mathrm{Ag} / \mathrm{AgCl} \mathrm{RE}$ without exchange membrane.

It is worth to notice that PVB, PVC and IL-PVC REs exhibit quasi-Nernstian response. In particular, IL-PVC shows the closest behaviour to the DJ RE, with the smallest standard deviation. On the contrary, the slope obtained with the $\mathrm{Ag} / \mathrm{AgCl}$ $\mathrm{RE}$ without membrane is almost double of the slope of the DJ RE. This can be explained considering that the bare $\mathrm{Ag} / \mathrm{AgCl}$ $\mathrm{RE}$ is highly sensitive to the concentration of chloride ions in solution. Chlorides ions seem to have high permeability also in the agar membrane as the slopes obtained with this RE is double of the Nernstian value and its standard deviation is significantly large.

In order to confirm these hypotheses and better understand the behaviour of the different membranes, the variations of RE potential with increasing chloride concentration is also investigated. All results are given in Figure 3 for three replicas of each RE type. As expected, the potential of the planar RE without membranes greatly decreases with the addition of chlorides to the sample solution. This behaviour explains 


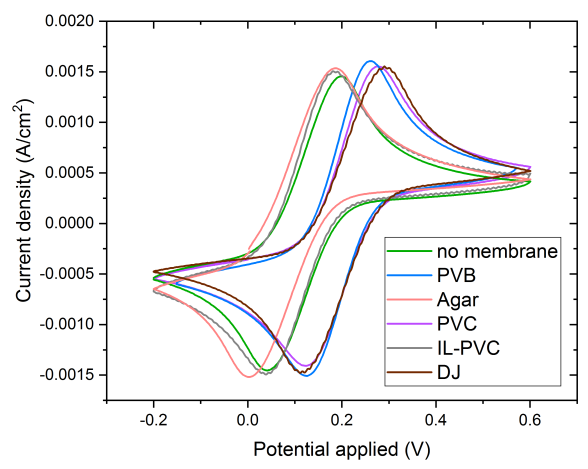

Fig. 6. Cyclic voltammograms of a gold screen-printed electrode in a $5 \mathrm{mM}$ potassium ferro/ferricyanide solution versus the different REs.

the high slope obtained during the calibration of the $\mathrm{Li}^{+}$ISEs.

The IL-PVC and PVB membranes have a limited drift until high concentrations are reached $\left(10^{-2}-10^{-1} \mathrm{M}\right)$. The PVC membranes shows the most stable potential at low concentration. Only at high concentration they start to exhibit a sensitivity to chloride ions. The lowest permeability of the PVC membranes is probably due to the more compact structure with respect to the other all-solid-state REs (Fig. 4). On the contrary, the different agar samples show significant drifts in both directions with limited reproducibility. This behaviour can be justified by considering the low elastic modulus of this material, that makes it very permeable and prone to detachments as evident in Figure 4.

The influence of $\mathrm{pH}$ on the RE potential was also analyzed. It is possible to observe from Figure 5 that in all cases the presence of a RE membrane drastically improves the stability at different $\mathrm{pH}$ and the reproducibility among different replicas. As for the previous tests, the agar membranes show the poorest behaviour. PVB, IL-PVC and PVC reference membranes drastically enhance the RE stability and reproducibility at different $\mathrm{pH}$.

The RE electrodes were finally tested for voltammetric applications. The cyclic voltammograms of a gold screen-printed electrode in a $5 \mathrm{mM}$ potassium ferro/ferricyanide solution are given in Figure 6 versus the different REs. All curves shows similar peak-to-peak distance to the DJ RE. The only excpetion is represented by the agar membranes which have a slightly higher values, corroborating the previous discussion on its poorer behaviour. The PVC and PVB membranes confirm their good performance, with an almost identical response to the $\mathrm{Ag} / \mathrm{AgCl} \mathrm{DJ} \mathrm{RE}$. The IL-PVC membranes have similar behaviour, but with a small shift $(0.1 \mathrm{~V})$ towards more negative potentials.

\section{CONCLUSION}

In this paper, we produce different REs membranes to characterize and compare their electrochemical behaviour in different conditions: agar, PVB, PVC, Ionic-Liquid-doped PVC. While agar offers only some minor improvements with respect to the bare $\mathrm{Ag} / \mathrm{AgCl} \mathrm{RE}$, all other membranes contribute significantly to the enhancement of the stability of allsolid-state Ag REs. The PVC RE shows the highest stability towards chlorides and $\mathrm{pH}$ variations, followed by $\mathrm{PVB}$ and IL-PVC. These differences can be explained by considering the different permeability of the membranes, as evident from the SEM analysis.

The membranes are then used to calibrate nanostructuredbased lithium ISEs. The PVB, PVC and IL-PVC membranes show quasi-Nenrstian behaviour. In particular, IL-PVC has a very similar value to the conventional DJ RE, with a small measurement error.

The PVC and PVB membranes confirms their good behaviour also for voltammetric applications, with almost identical responses to the DJ one. The IL-PVC also maintains the same peak-to-peak distance with a small shift of the all curve to more negative potentials $(0.1 \mathrm{~V})$.

In conclusion, PVB, PVC and IL-PVC membranes offer good stability and reproducibility with subtle differences depending on the application and on the $\mathrm{pH}$ of the working solution.

\section{ACKNOWLEDGMENT}

This research is supported by H2020 ERC 2014 ADG669354 CyberCare.

\section{REFERENCES}

[1] M. Sophocleous and J. K. Atkinson, "A review of screen-printed silver/silver chloride $(\mathrm{Ag} / \mathrm{AgCl})$ reference electrodes potentially suitable for environmental potentiometric sensors," Sensors and Actuators, A: Physical, vol. 267, pp. 106-120, 2017. [Online]. Available: http://dx.doi.org/10.1016/j.sna.2017.10.013

[2] U. Guth, "Solid-state reference electrodes for potentiometric sensors," Journal of Solid State Electrochemistry, no. January 2009, 2009.

[3] J. Hu, A. Stein, and P. Bühlmann, "Rational design of allsolid-state ion-selective electrodes and reference electrodes," Trends Anal. Chem., vol. 76, pp. 102-114, 2016. [Online]. Available: http://dx.doi.org/10.1016/j.trac.2015.11.004

[4] M. W. Shinwari, D. Zhitomirsky, I. A. Deen, P. R. Selvaganapathy, M. J. Deen, and D. Landheer, "Microfabricated Reference Electrodes and their Biosensing Applications," Sensors, no. 10, pp. 1679-1715, 2010.

[5] T. Zhang, C. Z. Lai, M. A. Fierke, A. Stein, and P. Bühlmann, "Advantages and limitations of reference electrodes with an ionic liquid junction and three-dimensionally ordered macroporous carbon as solid contact," Anal. Chem., vol. 84, no. 18, pp. 7771-7778, 2012.

[6] R. Maminska, A. Dybko, and W. Wr, "All-solid-state miniaturised planar reference electrodes based on ionic liquids," Sens. Actuators B, vol. 115, pp. 552-557, 2006.

[7] I.-y. Huang and R.-s. Huang, "Fabrication and characterization of a new planar solid-state reference electrode for ISFET sensors," vol. 406, pp. 255-261, 2002

[8] W.-y. Liao and T.-c. Chou, "Fabrication of a Planar-Form ScreenPrinted Solid Electrolyte Modified Ag / AgCl Reference Electrode for Application in a Potentiometric Biosensor," Anal Chem, vol. 78, no. 12, pp. 4219-4223, 2006.

[9] V. A. T. Dam, M. Goedbloed, and M. A. G. Zevenbergen, "Solid-Contact Reference Electrode for Ion-Selective Sensors," in Eurosensors Paris 2017, vol. 1 , no. 4, 2017, p. 464.

[10] T. Guinovart, G. A. Crespo, F. X. Rius, and F. J. Andrade, "A reference electrode based on polyvinyl butyral ( PVB ) polymer for decentralized chemical measurements," Analytica Chimica Acta, vol. 821, pp. 72-80, 2014. [Online]. Available: http://dx.doi.org/10.1016/j.aca.2014.02.028

[11] F. Criscuolo, I. Taurino, F. Stradolini, S. Carrara, and G. De Micheli, "Highly-stable Li + ion-selective electrode based on noble metal nanostructures as solid-contacts," Anal. Chim. Acta, 2018. 